\title{
A DECADAL ANALYSIS AND SENSITIVITY STUDY USING MOPITT CO COLUMNS OVER ASIA
}

\author{
Chuyong Lin ${ }^{1}$, Jason Cohen ${ }^{1 *}$ \\ ${ }^{1}$ School of Atmospheric Sciences, Sun Yat-Sen University, Zhuhai, 519082, China
}

KEY WORDS: Carbon Monoxide, pollution, MOPITT

\begin{abstract}
:
Biomass burning and urbanization are both significant sources of $\mathrm{CO}$ emissions and atmospheric loadings in the real environment. The sources of $\mathrm{CO}$ are due to incomplete combustion, on one hand of biomass from agriculture or forest clearing, and on the other hand from coal, oil, gas, and other similar materials. However, the spatial and temporal underlying properties and patterns are quite different between these two types of source regions, with urban regions having a relatively constant source of $\mathrm{CO}$ emissions, with only short term concentration fluctuations due to local meteorology. On the other hand, in biomass burning regions, the emissions themselves tend to be highly concentrated over a short burning period, and very low otherwise. We hence present a new technique to classify and quantify biomass burning regions and urban regions based on an objective analysis of the CO total column measurements from the MOPITT satellite. By using all of the data from 2000-2016, in connection with averages and standard deviation cutoffs, we successfully determine these regions. By performing a sensitivity analysis, in connection with additional ground-based measurements, we determine that the ideal cutoffs for the mean column loading and standard deviation of the column loading $28 \times 10^{17} \mathrm{~mol} / \mathrm{cm}^{2}$ and $6 \times 10^{17} \mathrm{~mol} / \mathrm{cm}^{2}$ respectively. These results are capable of representing known urban regions and biomass burning regions well throughout China, Southeast Asia, and South Asia, specifically including Beijing, Hebei, Shandong, Jiangsu, Anhui, Hunan, Guangdong, and Bangkok on one hand, and Northeastern India, Myanmar, Laos, Northern Thailand, and Vietnam on the other hand. A detailed analysis of the time series over the different classified regions show that while the urban areas have a much higher annual value, and a relatively long peak time, that their maximum is never as high as the peaks in the biomass burning regions, and that these peaks in the biomass burning regions are extremely short in duration, although they occur annually or bi-annually. Finally, we have not been able to obtain a statistically relevant decreasing trend, as others have found, making CO possibly an interesting species for future studies.
\end{abstract}

\section{INTRODUCTION}

Asia is presently one of the largest source regions for air pollution from both biomass burning and urbanization (Cohen and Wang, 2014; Cohen and Prinn, 2011). Biomass burning occurs in almost all areas of the world during the local dry season, particularly so in both rapidly developing countries, as well as those with intense agriculture (van der Werf et al., 2006). Additionally, urbanization is rapidly increasing through most developing countries. Asia, due to its rapid development, high population density, and intensive agriculture, has been increasing in terms of both of these sources over the past two decades (Cohen and Lecoeur, 2015).

Due to the length of the dry-season, meteorology, whether or not virgin forest is being cleared or accidently burned, and traditional farming techniques, there tend to be vast differences in the length and strength of biomass burning sources (Wang, 2017; Hansen et al., 2008). Some fires can occur pretty-much at random, in terms of time of the year and geography. This is because those people who live in the countryside or in areas without access to sufficient energy need to burn a significant amount of biomass for daily life, like cooking, light, heat, and clearing of rubbish. However, most tend to be seasonal in nature. This is because large-scale agricultural clearing or virgin forest removal tend to be seasonal in nature, occurring near the latter part of the local dry season or seasons (Dennis, 2005; Tipayarom and Oanh, 2007).

On the other hand, urban sources tend to be relatively steady and random throughout the year. Cities have economic activities that run throughout the year, with the other major sources of power generation and transportation tending to be relatively unimpacted by seasonal changes (Kurokawa et al., 2013; Zheng et al., 2014). While there has been definitive growth in the urban populations and formation of new cities, those areas which are urban tend to be relatively steady and stable on a week-to-week or month-to-month basis.
Both biomass burning and urban emissions are major sources of $\mathrm{CO}$, aerosols, heat, and other trace gasses, which are co-emitted together due to the incomplete combustion of the source material. While it is true that different sources and land surfaces have different ratios, all combustion sources will produce each of these species. Hence, since it is not water-soluble and not adsorbed by clouds, we will focus on the CO loadings as a proxy to study the major sources of biomass burning and urbanization.

Therefore, any short-to-moderate changes over time (shorter than a year) in the CO column loadings are associated with short-tomoderate time changes in CO emissions (Cohen and Prinn, 2011; Seinfeld and Pandis, 2006). On the other hand, long-term changes in CO column loadings (years to decades) are associated with both large-scale emission changes as well as the chemical changes associated with methane and $\mathrm{OH}$, the latter of which will not be further discussed in this work (Prinn et al., 1987; Prinn et al., 2001; Müller et al., 2018). When these perturbations/rapid changes are found to be small and random, occurring on the scale of a week or less, they tend to be similar to what is found in urban regions. On the other hand, when the perturbations are found to be large and seasonal (more than 1 week, and less than a couple of months), especially when they recur year-to-year, then they are consistent with biomass burning sources. This basic assumption is what forms the basis of this work.

\section{DATA AND METHODS}

In this paper, we use remotely sensed measurements from two different perspectives. From space, looking downward, we use the Measurements of Pollution in the Troposphere [MOPITT] instrument flying on the TERRA satellite, which gives us measurements of the CO total column loading (Deeter et al., 2014). On the ground, we use NASA's AERONET optical depth

*correspondence to Jason Cohen at jasonbc@alum.mit.edu 
measurements, specifically from the Dongsha Island and Lulin Mountain sites, which are both located in clean locations, yet in the outflow from the biomass burning regions (Dubovik et al., 2000; Cohen, 2014). We also use measured concentrations of CO from a network of 9 surface stations in Wuxi, which is a rapidly developing city located in the highly urban Nanjing to Shanghai corridor in mainland China (doi:10.6084/m9.figshare.7243460 ; Cohen and Wang, 2014).

Given the fact that there have been rapid changes in terms of urban development, agriculture, and clearing of primary forest occurring throughout Asia, we have chosen to focus our study on this area. In specific, we will focus on the area comprising the highly and densely populated regions of South Asia, Southeast Asia, and East Asia, specifically over the domain from $10^{\circ} \mathrm{N}$ to $55^{\circ} \mathrm{N}$ and from $70^{\circ} \mathrm{E}$ to $137^{\circ} \mathrm{E}$.

\subsection{MOPITT CO Total Column Measurements}

MOPITT is an instrument aboard the NASA Terra satellite, which has been collecting data from March 2000 through the present. In this paper, we use the $\mathrm{CO}$ total column, version 7 , Level 2. The mean and standard deviation of these measurements are given in Figures 1a and 1b, and have average values of $21.12 \times 10^{17} \mathrm{~mol} / \mathrm{cm}^{2}$ and $3.48 \times 10^{17} \mathrm{~mol} / \mathrm{cm}^{2}$ respectively over the climatology and the entire domain. For the purposes of this work, we take all measurements at the daily level and average them together into weekly measurements. The reason for this is that it provides us with solid statistics, since each individual missing pixel due to cloud contamination or in between swath gap is therefore removed from our data record or otherwise interpolated.
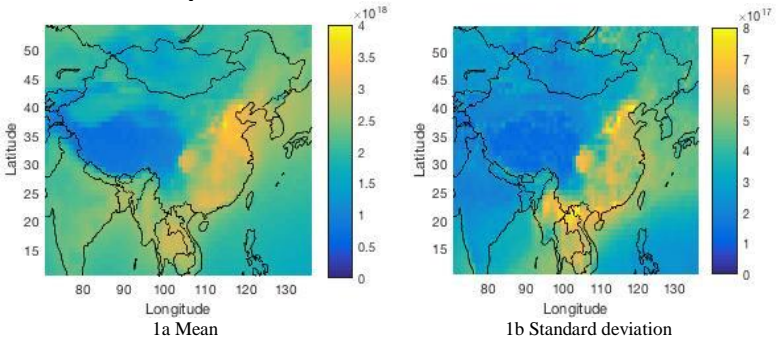

Figures 1a/1b. The climatological mean (1a) and climatological standard deviation (1b) of the MOPITT CO total column weekly measurements from 2000 through 2016.

\subsection{AERONET AOD and CO Concentration Measurements}

We specifically have obtained data from the Dongsha Island and Lulin Mountain stations of the AERONET network (version 3, all points data, Level 2). We then cloud-screen the data and make a weekly average with the all points data. The data from the stations is given in Figure 2. As can be observed, they are very similar to each other, with values typically very clean and low (under 0.2), except for during the biomass burning season, in which case values range from 0.5 to 1.5 . This similarity extends into the seasonal and temporal frequency and occurrence of the maximum. This fact is actually to be expected, since they are both found to be in relatively remote regions without too many local sources, while at the same time being in the outflow region far downwind from the same biomass burning sources in Southeast Asia, which occur each January through March. In general, Dongsha has a longer high period, in part because of the heavy cloudiness, in part because it is closer to the biomass burning regions, in part because it includes both boundary layer and free tropospheric air, and in part because of local shipping sources during certain times of the year. For this reason, we will use the data from both sites, but more likely rely on the data from Lulin Mountain in this analysis.

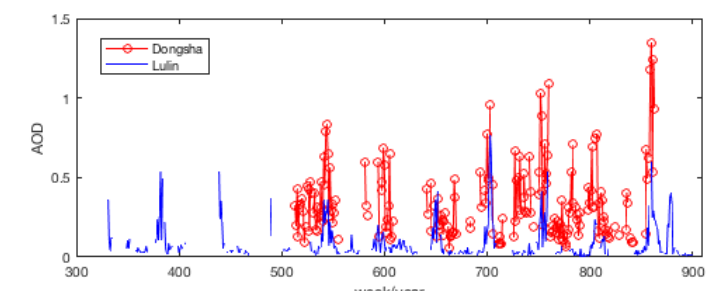

Figure 2. Measured AOD from the NASA AERONET network stations at Lulin (blue line) and Dongsha Island (red circles)

In addition, we have received hourly surface measurements of CO from ground stations in Wuxi, China, a rapidly urbanizing city located in the major Nanjing to Shanghai corridor. This city has been the location of an AERONET site for many years, and hence was chosen both because it is highly representative of an urban site, and because of additional international network measurements by which to validate the ground measurements. The data underlying these measurements can be found at (10.6084/m9.figshare.7243460). As can be observed in Figure 3, the data is highly noisy at hour-to-hour and day-to-day scales. However, on a weekly average, although the values are much higher than the NOAA GMD data above, the statistics show it is much less noisy in a relative sense, with a mean of $1.5 \mathrm{ppm}$ and a standard deviation of $0.57 \mathrm{ppm}$. This is because as an urban area, the local sources of $\mathrm{CO}$ tend to be relatively stable from week-toweek, and month-to-month, with most of the changes either due to local meteorological changes, or due to large-scale, seasonal atmospheric chemical changes in the lifetime of $\mathrm{CO}$ due to $\mathrm{OH}$ and the season of the year.

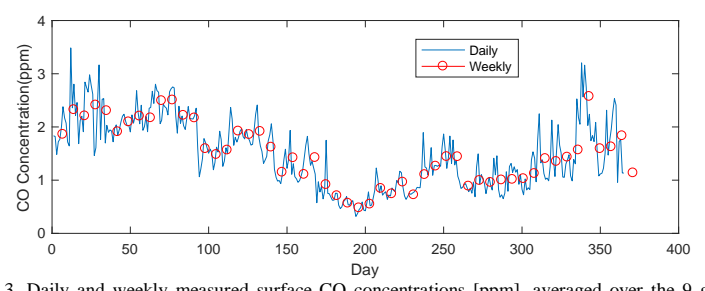
Figure 3. Daily and weekly measured surface CO concentrations [ppm], averaged over the 9 ground
stations in Wuxi, China, for the entire year of 2013. Data can be found at $10.6084 / \mathrm{m} 9$.figshare. 7243460

\subsection{Methods}

The seasonal burning of agriculture and the clearing of virgin forest both occur in the latter part of the dry season, after either the harvest is done, or when the trees have a relatively low moisture value. While it is true that different sources of biomass burning will have a different emissions ratio character, they all will co-emit $\mathrm{CO}$ and aerosols, with the contribution to the total atmospheric emissions in relatively clean regions in which biomass burning occurs being relatively powerful over such a short duration, on order of a week to a few weeks. This is very different from the urban measurements as shown above, which tend to be very noisy on an hourly to daily timescale, but which do not have a lot of variation on the weekly to multi-weekly time scale. Furthermore, urban areas tend to have their emissions roughly constant throughout the year, while in biomass burning regions, a significant fraction of their total annual emissions occurs during only a short time, and that this pattern tends to recur annually, although possibly with a slightly different strength or peak time from year to year. Seasonal emissions as found in biomass burning regions lead to a high variation, and a low yearly average, while on contrast, urban emission lead to a high yearly average and a low variation.

We employ different cutoff values for the average value, herein represented as the climatological mean, and the variation, herein represented as the climatological standard deviation. Therefore, since a precise cutoff is exact, it is essential for us to perform 
sensitivity tests to see how changes in the two cutoff values, the mean and the standard deviation, will relate to changes in the spatial and geographical extent on one hand, and the time series in the other hand, over the various biomass burning and urban regions. Therefore, we use the following facts to assist us: first that in the biomass burning regions, we expect to have an annual peak which is higher than in the urban regions, but an annual mean which is significantly lower. Secondly, we expect that the regions of Dongsha and Lulin on one hand, and Nanjing, Wuxi, and Shanghai on the other hand, must respectively be in the biomass and urban regions. Third, we expect that known remote and urban areas should be roughly classified correctly based on geography. If all of these conditions are not met then we have not been able to "ground-truth" the results. Thus, we will find a range and threshold for the variation and average, to identify the biomass burning emission region and urban emission region.

\section{RESULTS}

\subsection{Spatial-Temporal Distribution of the total CO Column}

The mean of the 17 years of weekly CO column measurements, from 2000-2016 is given in (fig 1a). The value of this statistic helps us to clearly identify which regions on average are producing the most amount of pollution. Polluted areas which are not substantially changing are well recognized over Eastern and Southeastern China, include: Jingjinji (Beijing, Hebei, and Tianjin) through Shandong, Henan, Shanghai, Anhui, Hubei, Hunan, Jiangxi, Guangdong, Guangxi, and Chengdu, as well as in central Thailand. Regions which have a very low mean are known to be clean, and are found mostly in Northwestern China, the Tibetan Plateau, and Mongolia.

On the other hand, the variance statistics of the 17 years of weekly CO column measurements, from 2000-2016 is given in (fig 1b). This value helps us to understand regions that are changing. In this case, we are interested in looking at changes induced by biomass burning, and as such we are looking at recurring temporary events, such as fires associated with agriculture or virgin forest conversion. It is also possible for rare meteorological events to have an impact, but we expect this to be negligible over such a long time-series of data which is nearly two decades in length. In particular, we are interested to separate those regions with a high variation and a relatively low mean, from those with a relatively low variation and a relatively high mean. Most of the former areas are relatively clean throughout the year, except for when the biomass burning occurs, such as Myanmar through Northern Thailand, Laos, and Northern Vietnam (excluding Hanoi). Only two locations within these regions are found to have an urban characteristic: the Pearl River Delta and Hanoi, both of which have been shown to be downwind recipients of biomass burning events (Lan and Cohen, et al., 2018, Lin et al., 2014; Lin 2016). The reason for this characteristic is that these regions are generally urban in their characteristics, but during the same time of year annually, experience biomass burning advected in from upwind. These specific regions will not be closely investigated further in this work, due to their complexity and uniqueness globally in terms of how their local $\mathrm{CO}$ measurements behave.

Every March, the CO total column over the northern parts of Southeast Asia reaches its annual maxima, which actually is also the monthly maxima for any region analysed in this study (Figure 4). This is quite unusual, since this region is otherwise cleaner than other regions throughout the domain studied here. While the local maxima is found in March, this entire region is found to have a "non-clean" value extending from sometime in January through sometime in April every year. Although the start and end times of this polluted region do vary from year to year. We know that this corresponds with the latter part of the dry season that occurs here annually, and is when both agricultural burning and forest conversion occur.

Due to the fact that the region with the biomass burning has an extremely high peak, yet a very low annual mean respectively, initial cutoffs for determining which region is which need to take this into account. We therefore set a cutoff for the variance that allows us to reproduce both the March annual peak, as well as the cleaner times occurring throughout most of the remainder of the year. On the other hand, we need to determine an annual mean CO column loading cutoff that is sufficiently large as to ensure we include the known urban regions in China and in Southeast Asia, such as around Bangkok. The minimum such cutoff to establish known urban regions is $24 \times 10^{17} \mathrm{~mol} / \mathrm{cm}^{2}$.
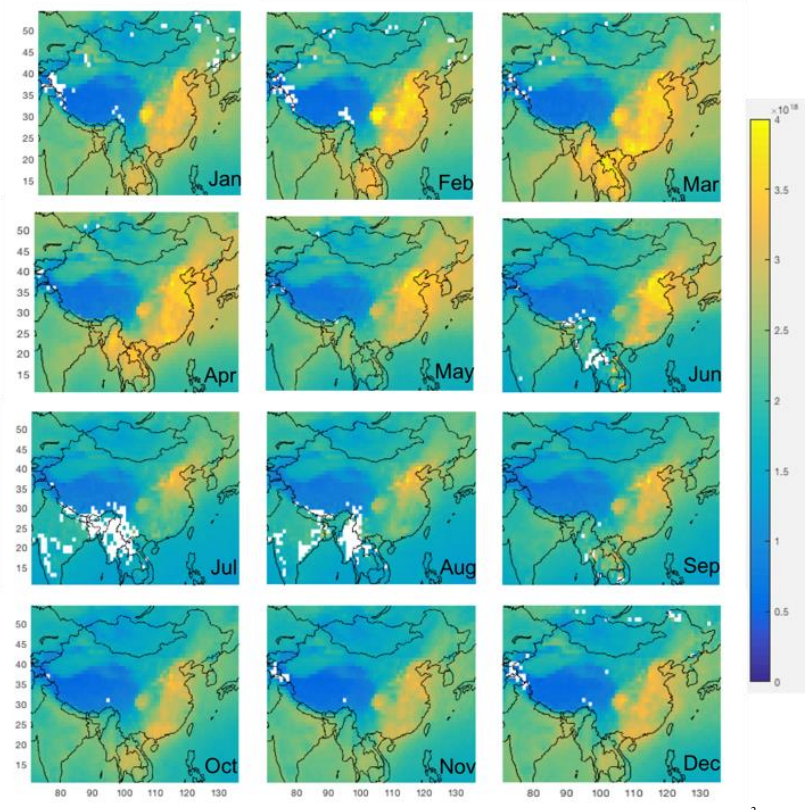

Figure 4: Month-by-month mean values of the CO total column over our region of interest $\left(\mathrm{mol} / \mathrm{cm}^{2}\right)$.

To determine the standard deviation cutoff over the biomass burning region, we first analyze only the data corresponding to the polluted period of the year (Figure 4). Therefore, to ensure that in fact our standard deviation cutoff when combined with our mean cutoff is able to capture the difference between the high and low CO column loading times, we first apply a filter to the biomass burning region data. We specifically filter the low data time as corresponding to $20 \%$ more than the annual average value over the same region. We then compute the annual average over the filtered "peak times" subset, and compute the cutoff standard deviation cutoff as two times this corresponding difference between the annual and "peak times" subset, which has a value of $5.4 \times 10^{17} \mathrm{~mol} / \mathrm{cm}^{2}$. This means that our cutoff minimum value already corresponds to a case where the polluted time period is at least 2 standard deviations above the non-polluted time period mean $\mathrm{CO}$ column loading value.

\subsection{Sensitivity Tests of the Mean and Standard Deviation Cutoff Values}

Since we have defined the minimum possible mean cutoff value to define the urban areas, we want to test and analyze how increasing this value will impact the ability for us to still represent known urban areas. In specific, we will test this by performing domain re-calculations a range of cutoffs applied to 
the mean and variance of the $\mathrm{CO}$ column loading. We start with a minimum mean CO column loading value of $24 \times 10^{17} \mathrm{~mol} / \mathrm{cm}^{2}$ and increase step-wise by $2 \times 10^{17} \mathrm{~mol} / \mathrm{cm}^{2}$ up to a maximum of $32 \times 10^{17} \mathrm{~mol} / \mathrm{cm}^{2}$. At the same time, we also will test how increasing the value of the minimum cutoff value of the $\mathrm{CO}$ column loading variance, associated with biomass burning areas will also impact the ability for us to still represent known biomass burning areas. In specific, we will test this by performing domain re-calculations using the known minimum cutoff in the standard deviation of the CO column concentration of $5.4 \times 10^{17} \mathrm{~mol} / \mathrm{cm}^{2}$ in increasing by steps of $2 \times 10^{17} \mathrm{~mol} / \mathrm{cm}^{2}$ up to a maximum value of $6.6 \times 10^{17} \mathrm{~mol} / \mathrm{cm}^{2}$.

The results of the re-computation of the various urban and biomass burning distribution maps are given in figure 5 . The plots have been arranged top-to-bottom in terms of the mean CO column cutoff values, with the lowest value being on top and the highest value being on the bottom. The maps are also correspondingly arranged from left-to-right in terms of the standard deviation of the $\mathrm{CO}$ column cutoff values, with the lowest value being on the left and the highest value on the right. The regions corresponding to urban are given in yellow, while the regions corresponding to biomass burning are given in red.

We observe spatially that the lower values of the CO total column cutoff yield vast areas of land which are not observed to have urban characteristics. For example, there are regions of Myanmar, Guangxi, and North Korea which are in these regions. However, we also observe that at very high values of the $\mathrm{CO}$ total column cutoff, many known urban areas are missing from the analysis, including Chongqing, Chengdu, Shanghai, Hangzhou, and Bangkok. This will lend towards the answer that a cutoff value near to either extreme is not so likely to be realistic.

However, to make the observations quantitative, we make a comparison between the ability of the time series of the weekly $\mathrm{CO}$ total columns measured by MOPITT over the region defined as "urban" to successfully reproduce the measured CO time series from surface $\mathrm{CO}$ concentrations in the urban area of Wuxi as given in Figure 3. Computing the correlation coefficient between the average $\mathrm{CO}$ column loading over each urban region, and the $\mathrm{CO}$ surface concentrations in Wuxi yields the observation that each of the three lower thresholds is similarly capable in terms of best reproducing the weekly average mean and maximum values as observed at Wuxi $\left(\mathrm{R}^{2}=0.72\right.$ for the $24 \times 10^{17}$ $\mathrm{mol} / \mathrm{cm}^{2}$ cutoff, $\mathrm{R}^{2}=0.71$ for the $26 \times 10^{17} \mathrm{~mol} / \mathrm{cm}^{2}$ cutoff, and $\mathrm{R}^{2}=0.72$ for the $28 \times 10^{17} \mathrm{~mol} / \mathrm{cm}^{2}$ cutoff). On the other hand, the two highest cutoff values have a decreasing ability to reproduce the measured $\mathrm{CO}$ surface values in Wuxi, with a respective value of $\left(\mathrm{R}^{2}=0.63\right.$ for the $30 \times 10^{17} \mathrm{~mol} / \mathrm{cm}^{2}$ cutoff, and $\mathrm{R}^{2}=0.58$ for the $32 \times 10^{17} \mathrm{~mol} / \mathrm{cm}^{2}$ cutoff) (Figure 6). A comparison between the three lower cutoff cases shows that the major difference is found in terms of a strong decrease in the mean CO column loading itself, as compared with the ability to reproduce different loadings over time. This is due to the fact that many "relatively clean" areas are incorporated into the urban definition at the lower values. Therefore, we make the argument that the best urban cutoff is represented by $28 \times 10^{17} \mathrm{~mol} / \mathrm{cm}^{2}$.

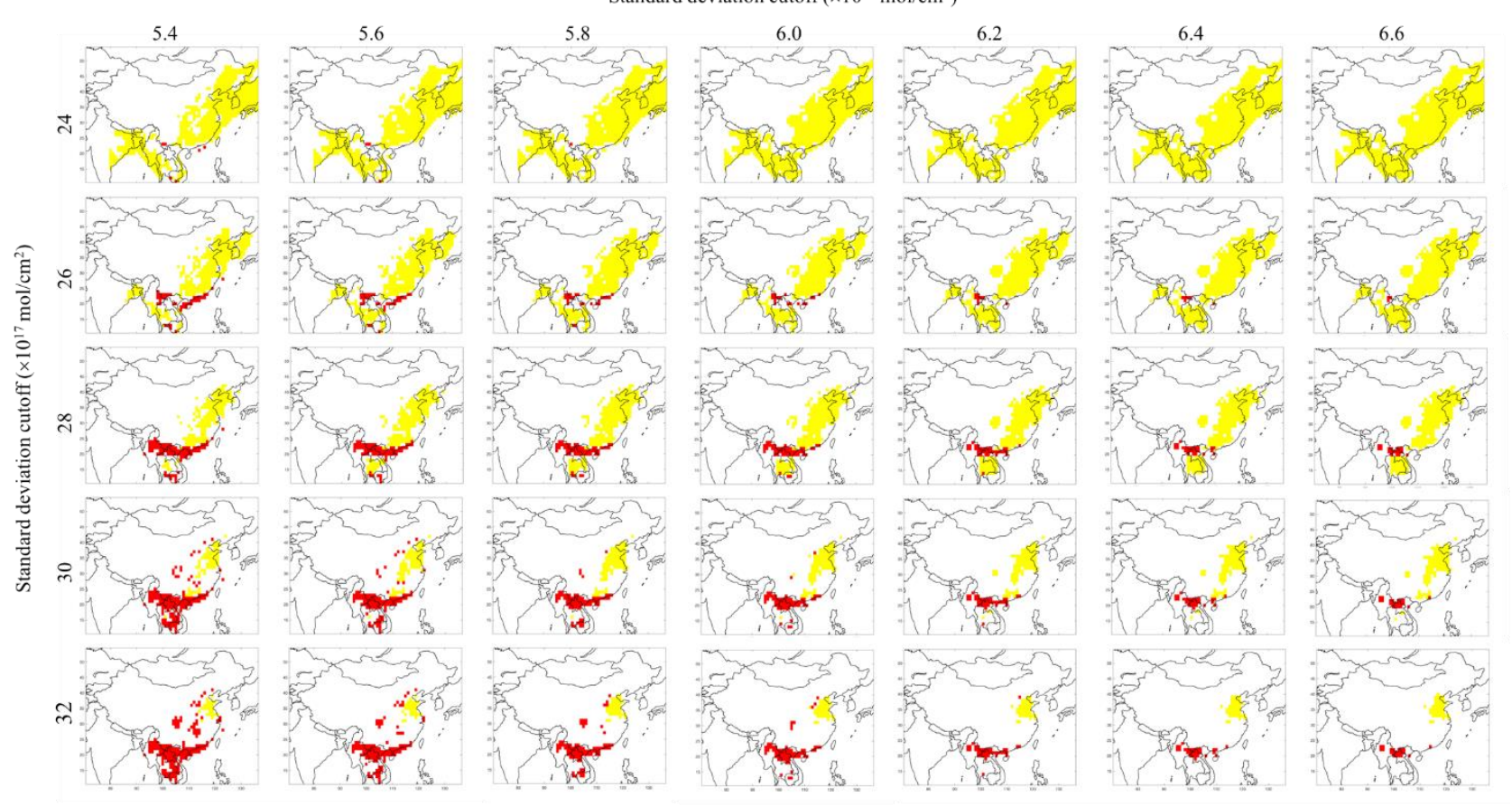

Figure 5. Maps of urban regions (yellow) and biomass burning regions (red), using different cutoff values for CO column mean (changes vertically from top to bottom corresponding respectively to a CO column concentration of $24 \times 10^{17} \mathrm{~mol} / \mathrm{cm}^{2}$ through $32 \times 10^{17} \mathrm{~mol} / \mathrm{cm}^{2}$ ) and CO column standard deviation (changes horizontally from left to right, corresponding respectively to a CO column standard deviation of $5.4 \times 10^{17}$ $\mathrm{mol} / \mathrm{cm}^{2}$ through $6.6 \times 10^{17} \mathrm{~mol} / \mathrm{cm}^{2}$ ). 


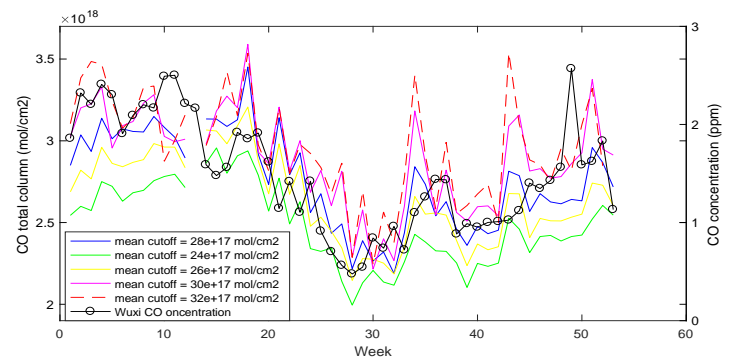

Figure 6. Measured surface CO [ppm] from Wuxi, only available for 2013, is given in black circles on the right-hand axis. $\mathrm{CO}$ total columns averaged over the various urban regions being tested, only using data from 2013, are given on the left-hand axis, where the [red dashes] is the $32 \times 10^{17} \mathrm{~mol} / \mathrm{cm}^{2}$ cutoff, the [magenta line] is the $30 \times 10^{17} \mathrm{~mol} / \mathrm{cm}^{2}$ cutoff, the [blue line] is the $28 \times 10^{17} \mathrm{~mol} / \mathrm{cm}^{2}$ cutoff, and the [green line] is the $24 \times 10^{17} \mathrm{~mol} / \mathrm{cm}^{2}$ cutoff. In all cases, a standard deviation cutoff of $6.0 \times 10^{17} \mathrm{~mol} / \mathrm{cm}^{2}$ was applied.

We observe spatially that the lower values of the CO standard deviation cutoff yield vast areas downwind from known burning areas, including most of coastal Southern China. While this area does have small amounts of biomass burning, there is definitely no known such large-scale effect here. And while there is some work indicating that this region is in the downwind outflow from Southeast Asian biomass burning, to have such an extensive region over land is not necessarily consistent with the measurements, that show the plume to extend only a small fraction of the total dry-season days. On the other hand, at very high CO standard deviation cutoff values, the very small amount of burning regions vastly underestimates fire hotspots which are observed in Thailand, Cambodia, Vietnam, and Myanmar. Given that $\mathrm{CO}$ can rise above clouds and travel downwind, we should not have a case in which the $\mathrm{CO}$ measurements are less geographically diverse than the hotspot measurements are themselves.

However, to make the observations quantitative, we make a comparison between the ability of the time series of the weekly CO total columns measured by MOPITT to successfully reproduce the high and low peaks of measured $\mathrm{CO}$ from the NASA AERONET measurements given at Dongsha Island and Lulin Mountain as given in Figure 2.

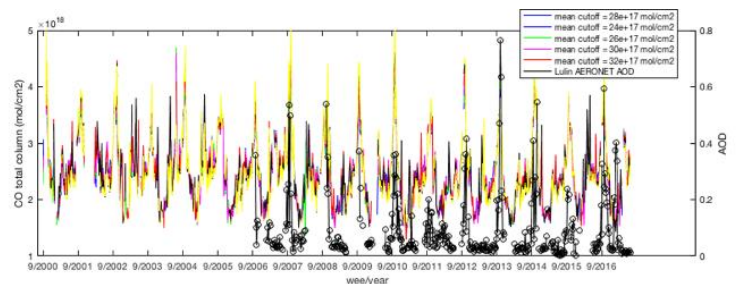

Figure 7. Measured AERONET AOD [circles] from Lulin Mountain, and CO total column mean value over various biomass burning regions with cutoffs as given in the plot.

Computing the correlation coefficient between the average $\mathrm{CO}$ column loading over each urban region, and the AERONET AOD values at both Dongsha Island and Lulin Mountain yield results which are identical for each of the cases. This is because each case is capable of reproducing the far-downwind transport which impacts the high AOD conditions at these sites. This is when we match the maximum to each other.

However, another way of looking at this can be from the perspective of the statistics: what are the mean and standard deviation of the various cutoff biomass burning $\mathrm{CO}$ total column average time series, and how do the mean and standard deviation compare with the same as computed from the AERONET data at Dongsha island and Lulin Mountain. The ratio of the standard deviation to the mean at Lulin is found to be 1.27 , while the ratio of the standard deviation to the mean is found to be 0.68 .
In reality, while the $\mathrm{CO}$ total columns have a standard deviation to mean ratio which is larger than Dongsha, it is also smaller than Lulin for all cases. Therefore, the important point to consider is what is physically possible, given the current data is not sufficient to differentiate between the cases. Hence, the selection needs to include at a minimum the regions which have fire hotspots (val Martin et al., 2018), and at a maximum, which are found within the region given by Lan, Cohen, et al. (2018) Hence, we can restrict the end results can range anywhere from $5.8 \times 10^{17}$ $\mathrm{mol} / \mathrm{cm}^{2}$ to $6.2 \times 10^{17} \mathrm{~mol} / \mathrm{cm}^{2}$.

With these thresholds now established, we can say with a degree of certainty, as well as describe what regions are most likely to change. In the end, our best choice for the urban cutoff leads us to a definition that includes most of the truly urban regions in this region of the world, including: Beijing, Tianjin, Hebei, Shandong, Jiangsu, Anhui, Hunan, Guangdong, Chengdu, Chongqing, and Bangkok. On the other hand, our definition of biomass burning leads us to a region that describes most of the regions which are known to be undergoing biomass burning throughout this region. This includes the arc along the Southern side of the Himalayas extending from Northeastern India, through Myanmar, Northern Thailand, Laos, and Vietnam. It also includes a small amount of known rice-straw burning in Central Thailand and Cambodia.

\subsection{Analysis of the Similarities and Differences Between the Time Series of Urban and Biomass Burning Regions}

The time series of the weekly mean of CO total column over both the urban region and the biomass burning (using cutoffs of $28 \times 10^{17} \mathrm{~mol} / \mathrm{cm}^{2}$ and $6.0 \times 10^{17} \mathrm{~mol} / \mathrm{cm}^{2}$ ) from 2000 through 2016 are given in Figure 8. As can be observed from the statistics, the urban region has an average value of $31.1 \times 10^{17} \mathrm{~mol} / \mathrm{cm}^{2}$ and a standard deviation of values of $3.7 \times 10^{17} \mathrm{~mol} / \mathrm{cm}^{2}$, while the biomass burning region has an average value of $24.9 \times 10^{17}$ $\mathrm{mol} / \mathrm{cm}^{2}$, and a standard deviation of $5.8 \times 10^{17} \mathrm{~mol} / \mathrm{cm}^{2}$. These results are consistent with the assumptions and findings presented above in terms of the magnitudes and extreme values expected to be observed in the total CO column between these two different subregions.

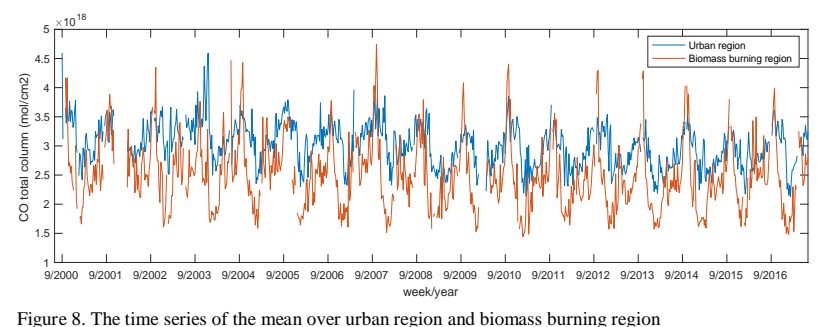

While both of these regions have some oscillation over the course of the year, the oscillation in the urban regions is much smaller. This is even though there is significant secondary $\mathrm{CO}$ production chemically occurring in these regions due to oxidation of VOCs. However, this is mostly because of the emissions being roughly regular throughout the year. There is also the impact of the global $\mathrm{OH}$ changes and other atmospheric oxidation changes that occur as a function of the solar flux. This helps to explain why both regions have an annual trough and low point through the most intense months of the summer. Although it is obvious to observe that the biomass burning regions are much lower than over the urban regions. It is also observed that both regions have a peak in the cooler and darker times of the year. However, the peak over the urban regions seems to be quite broad and spread out, with it occurring throughout the period from November through March, while in the biomass burning regions, the peak tends to be very 
tight/short in duration, at most lasting a few weeks, and always occurs in February or March. Also, it is important to point out that the peaks at the height of the biomass burning season are much higher than the peaks in the urban areas as well.

In some years, the biomass burning regions have a second peak in September or October. This is consistent with the dual nature of the Monsoon in this region of the world. In these years where there is a sufficiently dry second season, frequently the straw from a second rice harvest will be burned, or additional land clearing will be made.

It is also very interesting to note that while many other studies are reporting a definitive drop in Asian air pollution, in terms of long-term trends, we cannot find this in our work. Perhaps $\mathrm{CO}$ is not decreasing statistically along with other co-emitted species. However, we also are very careful in terms of our statistics, and given the high amount of uncertainty in the measurements and the methods being applied, as well as the high level of variability of the time series, we find that the uncertainty is too high for any meaningful statistics to be obtained in terms of trends. However, in terms of extremes, we feel much more comfortable about our results.

\section{DISCUSSION AND CONCLUSIONS}

First of all, we have successfully analyzed the entire subset of daily MOPITT CO total column measurements from 2000 through 2016. By applying metrics of the mean and standard deviation to the weekly data over our area of interest in South Asia, Southeast Asia, and East Asia, we have been able to derive a classification of Urban and Biomass Burning regions. The results show us that the urban types of regions are mostly found in Eastern China and Southeastern China, with additional pockets in and around Chengdu/Chongqing and Bangkok. In specific, the urban classification includes the known urban regions of Jingjinji, Shandong, Henan, Shanghai, Jiangsu, Zhejiang, Anhui, Hubei, Hunan, Jiangxi, Guangdong, as well as Chengdu, Chongqing, and in central coastal Thailand. Furthermore, this approach has allowed us to classify biomass burning regions. These are regions which have a high variation occurring over a short duration of time, and an either annual or bi-annual peak. In general, these areas are quite clean except for a short period of time from January through April, with the peak almost exclusively occurring in March. These areas are also quite precise in terms of where they are located, and include the swath along the Southern side of the Himalayas from Northeastern India through Myanmar, Northern Thailand, Laos, and Vietnam. There is also a small area in Eastern Thailand and Cambodia that corresponds to known agricultural burning there.

Secondly, we have conducted extensive testing of the sensitivity of the mean and standard deviation cutoff values from the MOPITT CO total column measurements. By carefully analysing step-wise changes in the cutoff of the $\mathrm{CO}$ total column mean, ranging from $24 \times 10^{17} \mathrm{~mol} / \mathrm{cm}^{2}$ to $32 \times 10^{17} \mathrm{~mol} / \mathrm{cm}^{2}$, and simultaneously in the cutoff of the CO total column standard deviation from $5.4 \times 10^{17} \mathrm{~mol} / \mathrm{cm}^{2}$ to $6.6 \times 10^{17} \mathrm{~mol} / \mathrm{cm}^{2}$, we are able to select which set of values gives us the ideal solution. We have used measurements of $\mathrm{CO}$ from various sites, and knowledge of geography to help constrain our ideal cutoff values. In the end, we conclude that the best combination of CO total column mean and $\mathrm{CO}$ total column standard deviation are given by $28 \times 10^{17} \mathrm{~mol} / \mathrm{cm}^{2}$ and $6 \times 10^{17} \mathrm{~mol} / \mathrm{cm}^{2}$ respectively. With these thresholds, we can observe that the urban regions and the biomass burning regions are both found as expected and explained above.
Thirdly, an analysis of the time series yields a few interesting points. One of these is that while the mean values are considerably higher in the urban regions than the biomass burning regions in general and throughout most of the year, there is a small but obvious period of time each year in which the biomass burning regions $\mathrm{CO}$ total column loading is higher than in the urban region. Furthermore, this time is always relatively short, but does not always exist for the same length of time, with the start and end times changing from year to year, but always at least partially encompassing March. Furthermore, we have not been able to determine a statistically relevant decreasing trend in the CO total column measurements over either the urban or the biomass burning region. Perhaps this is because the $\mathrm{CO}$ is not decreasing along with other pollution species, or perhaps because we are looking at the high frequency data.

\section{REFERENCES}

Cohen J B and Prinn R G, 2011. Development of a fast, urban chemistry metamodel for inclusion in global models, Atmos. Chem. Phys., 11, 7629-7656, https://doi.org/10.5194/acp-117629-2011

Cohen J B and Wang C, 2014. Estimating global black carbon emissions using a top-down Kalman Filter approach, J. Geophys. Res.-Atmos., 119, 307-323

Cohen J B 2014. Quantifying the occurrence and magnitude of the Southeast Asian fire climatology. Environmental Research Letters, 9(11), 114018. doi:10.1088/1748-9326/9/11/114018

Cohen J B 2018. CO hourly data from 9 stations in Wuxi China. DATASET. doi: 10.6084/m9.figshare. 7243460

Cohen J B, et al., 2017. Decadal-scale relationship between measurements of aerosols, land-use change, and fire over Southeast Asia, Atmospheric Chemistry and Physics, 17, 721743, doi:10.5194/acp-17-721-2017

Deeter M N, et al., 2014. The MOPITT Version 6 product: algorithm enhancements and validation. Atmospheric Measurement Technology, 7, 3623-3632, doi:10.5194/amt-73623-2014.

Dennis, et al., 2005. Fire, people and pixels: linking social science and remote sensing to understand underlying causes and impacts of fires in Indonesia, Hum. Ecol., 33, 465-504, doi:10.1007/s10745- 005-5156-Z

Dubovik O, et al., (2000) Accuracy assessments of aerosol optical properties retrieved from Aerosol Robotic Network (AERONET) Sun and sky radiance measurements. J. Geophys. Res., 105(D8), 9791-9806.

Hansen, et al., 2008. Hu- mid tropical forest clearing from 2000 to 2005 quantified by using multitemporal and multiresolution remotely sensed data, P. Natl. Acad. Sci. USA, 105, 9439-9444

Kurokawa J, 2013. Emissions of air pollutants and greenhouse gases over Asian regions during 2000-2008: Regional Emission inventory in Asia (REAS) version 2, Atmos. Chem. Phys., 13, 11019-11058, doi:10.5194/acp-13-11019-2013

Lan R Y, Cohen J.B., Lin C.Y., and Ng H L D. 2018. Combining Models and Measurements to Constrain Smoke Aerosols over the Asian Monsoon Region (UNDER REVIEW), Presented at the AGU 2017 Annual Meeting, USA. A110-07

Lin N H, 2016. Investigation of Biomass Burning Chemical Components over Northern Southeast Asia during 7SEAS/BASELInE 2014 Campaign. Aerosol and Air Quality Research. doi: 10.4209/aaqr.2016.03.0105 
Lin $\mathrm{N} \mathrm{H}$, et al., 2014. Interactions between biomass-burning aerosols and clouds over Southeast Asia: Current status, challenges, and perspectives. Environmental pollution, 195, 292307. doi: 10.1016/j.envpol.2014.06.036

Müller J F, et al. 2018. Top - down CO emissions based on IASI observations and hemispheric constraints on $\mathrm{OH}$ levels. Geophysical Research Letters, 45, 1621 - 1629. https://doi.org/10.1002/2017GL076697

Prinn R, et al., 1987. Atmospheric Trends in Methylchloroform and the Global Average for the Hydroxyl Radical. Science, $10.1126 /$ science. 238.4829 .945

Prinn R G, et al., 2001. Evidence for substantial variations of atmospheric hydroxyl radicals in the past two decades, Science, $292,1,882$.

Tipayarom D, et al., 2007. Effects from open rice straw burning emission on air quality in the Bangkok Metropolitan Region, Science Asia, 33, 339-345

val Martin M, Kahn R A, and Tosca M, 2018. A Global Climatology of Wildfire Smoke Injection Height Derived from Space-based Multi-angle Imaging. Remote Sensing 10, 1609; doi:10.3390/rs10101609.

van der Werf, et al., 2008. Climate regulation of fire emissions and deforestation in equatorial Asia, P. Natl. Acad. Sci. USA, 105, 20350-20355, doi:10.1073/pnas.0803375105

Wang C, 2007. Impact of direct radiative forcing of black carbon aerosols on tropical convective precipitation, Geophys. Res. Lett., 34, L05709, doi:10.1029/2006GL028416

Zheng B, et al., 2014. High-resolution mapping of vehicle emissions in China in 2008, Atmos. Chem. Phys., 14, 9787-9805, doi:10.5194/acp-14-9787-2014

Revised Oct 2018 